\title{
Photon subtraction from traveling fields - recent experimental demonstrations
}

\author{
Jonas S. NEERGAARD-NIELSEN ${ }^{1}$, Makoto TAKEUCHI ${ }^{2}$, Kentaro WAKUI ${ }^{3}$, Hi- \\ roki TAKAHASHI ${ }^{4}$, Kazuhiro HAYASAKA ${ }^{5}$, Masahiro TAKEOKA ${ }^{6}$, and Masahide \\ SASAKI \\ 1,2,3,5,6,7 National Institute of Information and Communications Technology (NICT) \\ ${ }^{4}$ University of Sussex
}

\begin{abstract}
We review our most recent results on application of the photon subtraction technique for optical quantum information processing primitives, in particular entanglement distillation and generation of squeezed qubit states. As an introduction we provide a brief summary of other experimental accomplishments in the field.
\end{abstract}

\section{KEYWORDS}

Quantum information processing, entanglement distillation, continuous variable qubit, coherent state quantum computing, squeezed light, photon subtraction, homodyne tomography

\section{Introduction}

For a number of years, our group has been working on generation, manipulation and detection of nonclassical quantum states of light using techniques and concepts from both the continuous variable (cv) and the discrete variable (dv) subfields of quantum information science. By doing so, we have taken part in a trend both theoretical and experimental — of moving away from seeing the cv and dv paradigms as separate, competing frameworks for quantum information processing and rather look for ways of taking advantage of and combining the best properties of both.

In this paper, we review our two most recent results in this direction: Entanglement distillation of Gaussian states [1] and generation of arbitrary qubit-like squeezed states [2]. These two experiments demonstrate some of the possibilities that arise when combining squeezed vacuum or other non-classical cv states with the technique of photon subtraction. Before getting to that, we give a short (and incomplete) overview of a number of results in quantum optics and quantum information that brought us to the current state of affairs.

Received November 1, 2010; Revised December 9, 2010; Accepted December 27, 2010.

1) neergaard@nict.go.jp, ${ }^{2)}$ takeuchi@phys.c.u-tokyo.ac.jp,

3)wakui@nict.go.jp, ${ }^{4)}$ H. Takahashi@sussex.ac.uk, ${ }^{5)}$ hayasaka@nict.go.jp,

6) m.takeoka@soumu.go.jp, ${ }^{7}$ psasaki@nict.go.jp

DOI: 10.2201/NiiPi.2011.8.1

\section{A brief history of bridges}

\subsection{Motivation}

When optical quantum information processing was developed through the 90's, a reasonably strong distinction was made between the two different "regimes" of discrete and continuous variable systems. This distinction persists to some extent even today. In fact there is no fundamental difference between the two; rather, they are complementary ways of describing the states of quantized electromagnetic fields [3].

Most quantum optics experiments and quantum information schemes consider information contained in discrete degrees of freedom of an optical field: photon numbers, orthogonal polarization or spatial modes, time bins and so on. The research of a number of groups, however, looks into states of light that are more conveniently expressed in terms of continuous degrees of freedom, typically as non-commuting observables like the phase and amplitude quadratures or Stokes polarization vectors. A typical state representation in $\mathrm{dv}$ is the density matrix over the available orthogonal modes of the system, while cv states are often represented by their Wigner functions - quasi-probability distributions in the phase space of conjugate variables. However, a corresponding density matrix can be straightforwardly calculated from a Wigner function and viceversa, so the choice of representation is just one of convenience, clarity and perhaps convention. 
The interest in using continuous variables for quantum information processing originates largely from the efficiency of various processes: Squeezed light and coherent states can be generated deterministically, homodyne detection can achieve almost $100 \%$ efficiency, and many standard operations are deterministic and easy to implement in the lab. Furthermore, all of these processes can be described in the simple and well understood Gaussian framework where states are represented solely by their means and covariance matrices. $\mathrm{Cv}$ quantum information processing is not limited to optical systems, but have also been explored in atomic systems such as spin-polarized ensembles of neutral atoms, and there are several promising approaches for efficiently implementing light-atom interactions [4]. Finally, it should be mentioned that modern optical communications uses quadrature encoding (cv) and therefore cv approaches to quantum information may yield better compatibility with existing infrastructure.

Experimentally, one of the driving forces behind optical implementations of quantum information primitives and protocols has been the process of parametric down-conversion. Although there exist protocols for quantum key distribution using only classical coherent states, for basically all other purposes a source of non-classical light is required, and for that, parametric down-conversion is one of the simplest and most efficient methods around. By a second-order nonlinear interaction in a crystal or waveguide, a single photon from a pump beam is converted into two daughter photons correlated in a number of different degrees of freedom. For weak pumping, this results in generation of photon pairs, often with orthogonal polarization (type II down-conversion) such that they are easily separable. For stronger pumping and degenerate downconverted photons, the single output beam has quantum noise fluctuation below the standard quantum limit and is said to be in a squeezed state. These two different aspects of the down-conversion output lend themselves to detection either by photon counting/single photon detection or by homodyne detection, respectively. Photon detection gives a naturally discrete measurement outcome, while homodyne detection provides a continuous-valued measurement of a given quadrature variable. While the photon generation and measurement process is probabilistic, squeezing generation and homodyne measurement is fully deterministic. This was reflected in the first experimental demonstrations of quantum teleportation by Bouwmeester et al. [5] (dv) and Furusawa et al. [6] (cv) - these almost simultaneous papers are perhaps the most prominent examples of the distinction between the two paradigms.

\subsection{Experiments}

In 2001, Lvovsky et al. [7] presented probably the first experiment to truly bridge the $\mathrm{dv}$ and $\mathrm{cv}$ worlds. They generated a single photon state by heralding on detection of one of the photons of a parametrically down-converted twin pair, and then measured its Wigner function by homodyne tomography (full state characterization by homodyne detection of a large ensemble of identically prepared states). They essentially measured the continuous-variable representation of an intrinsically discrete quantum state, the one-photon Fock state. Its Wigner function showed a prominent dip below zero at the phase space origin - a feature that is common to all non-Gaussian pure states, but that had not been observed in optics until then, since homodyne measurements had only been applied to Gaussian states such as squeezed vacuum or coherent states. This accomplishment was followed up by Babichev et al. [8] who performed two-mode tomography of a dualrail single photon, and by Zavatta et al. [9] who showed the Wigner functions of photon-added coherent states, obtained by seeding a coherent beam into one output of a non-degenerate parametric down-converter.

A later breakthrough in the merger of $d v$ and cv technologies came when Ourjoumtsev et al. [10], Neergaard-Nielsen et al. [11] and later Wakui et al. [12] combined squeezed vacuum generation with photon detection-heralded homodyne tomography to demonstrate small coherent state superposition states, also called Schrödinger kittens. Such states of the form $|\psi\rangle=|\alpha\rangle-|-\alpha\rangle$ will be useful for quantum information processing [13]-[16] but are unfortunately extremely difficult to prepare in a deterministic manner. Dakna et al. [17] suggested already in 1997 a way to probabilistically make high-fidelity approximations to these states for small coherent amplitudes $\alpha$, simply by subtracting one or more photons from squeezed vacuum. Wenger et al. [18] showed the first signs of non-Gaussian statistics from a state prepared in this way, and this was later followed up by the full negative Wigner function state preparations [10]-[12]. The photon subtraction operation itself has turned out to be a generally useful tool for quantum information processing and for fundamental tests of quantum mechanics, as explored in a series of theoretical studies [19]-[23].

These experiments originate in the $\mathrm{cv}$ world with squeezing and homodyne tomography, but incorporate photon detection and conditional state preparation from the $d v$ toolbox. In the measured output states, their dual nature is reflected in the Wigner function which is a mix between a single photon state and a squeezed vacuum (it is in fact a squeezed photon). We can therefore no longer claim convincingly that the generated states or the experiments as such belong to the $\mathrm{dv}$ or the $\mathrm{cv}$ 
regimes - rather they are bridging these two worlds. This trend of $\mathrm{dv} / \mathrm{cv}$ bridging experiments that slowly started with the results from Lvovsky and Bellini's labs in the first half of the decade has taken off dramatically in the last few years, especially since the first kitten generation demonstrations in 2006-7. To our knowledge, kitten states have now been observed in Paris, Copenhagen, three labs in Tokyo, and at NIST. Most recently, photon-number resolving transition edge sensors were used to prepare three-photon subtracted squeezed vacuum [24] and telecom wavelength twophoton subtracted squeezed vacuum [25].

Other interesting experiments that are worth mentioning are the two-photon Fock state tomography [26], the time-delayed two-photon subtraction for enlarging the even kitten state $|\alpha\rangle+|-\alpha\rangle$ [27], a squeezed kitten state prepared by conditional homodyning of a 2photon Fock state [28], remote preparation of entangled kitten states [29], a direct test of the commutation relations, confirmed by homodyne tomography [30], a tunable POVM detector combining homodyne detection with photon number resolution [31], tomography of photons from a pulse pumped OPO [32], tomography of 0-, 1-, 2-photon Fock state superpositions [33] and of intermediate single photon/squeezed vacuum states [34], noiseless amplification of weak coherent states by photon detection [35]-[38], photon counting-assisted optimal coherent state discrimination [39]-[41], and teleportation of a kitten state [42]. Kim [43] has also compiled a nice review of the theoretical foundations and experimental progress in this research area, while Lvovsky and Raymer [44] gives a comprehensive review focused on the methods and applications of $\mathrm{cv}$ state tomography. A more general overview of various dv/cv hybrid approaches to quantum information processing is provided by Van Loock [45].

\section{Experiment basics}

Both of the two experiments detailed later are based on our setup for generation of photon-subtracted squeezed vacuum (kitten state). We will here briefly go through some technical details of this experiment, so that we can better focus on the conceptual ideas later.

There are essentially three stages of the kitten generation: Resource state preparation (squeezed vacuum), state manipulation (photon subtraction) and state characterization (homodyne tomography). These are illustrated in Fig. 1 and described below in turn. For more details, refer to [12], [27].

\subsection{Squeezed vacuum}

Our source of non-classical light is a continuously pumped optical parametric oscillator (OPO) consisting of a $10 \mathrm{~mm}$ long periodically poled potassium titanyl

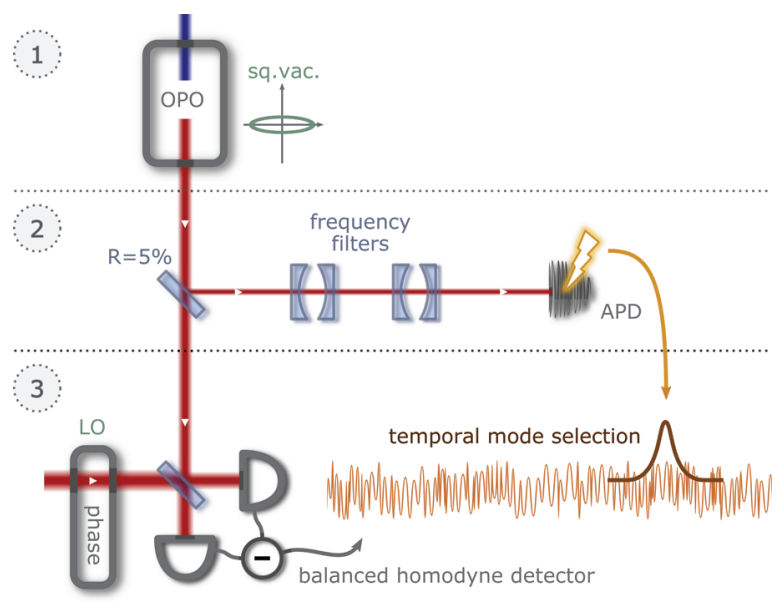

Fig. 1 Conceptual setup for generation of single-photon subtracted squeezed vacuum. 1) Squeezed vacuum preparation. 2) Photon subtraction. 3) Homodyne tomography.

phosphate (PPKTP) crystal in a bow-tie type singleended optical cavity. It is pumped by a frequencydoubled Ti:Sapph laser with pump wavelength $430 \mathrm{~nm}$. The cavity has a $580 \mathrm{MHz}$ free spectral range and the FWHM bandwidth is $9 \mathrm{MHz}$, and because of the low internal losses, the outcoupling efficiency is as high as $96 \%$, which leads to almost pure squeezing at the output. This OPO configuration has proven to be able to deliver up to $9 \mathrm{~dB}$ of squeezing [46], but high squeezing levels result in a higher degree of mixedness in the observed temporal modes because the spectral character of the state becomes "skewed"; the bandwidth of the squeezed quadrature is $\gamma+\epsilon$, while for the anti-squeezed quadrature it is $\gamma-\epsilon$, where $\gamma$ is the OPO bandwidth and $\epsilon$ the pump parameter [47], [48]. For this reason, we usually generate squeezing in the range $2-5 \mathrm{~dB}$.

In order to control the orientation of the squeezing in phase space, we inject a coherent probe beam through one of the high-reflectivity mirrors of the OPO. This beam then co-propagates with the squeezed vacuum and can be used for phase locking as well as locking of the filter cavities in the photon subtraction channels. For phase locking the squeezing orientation, we monitor the transmitted beam on a standard DC detector. The beam has undergone classical phase-dependent parametric amplification in the OPO, so by fixing the amount of amplification, the probe beam will be locked in phase with the squeezed vacuum. By later locking the probe beam to the local oscillator of the homodyne detector (which determines the phase space orientation), we can fix the squeezing orientation.

The probe beam is much stronger than the squeezed vacuum, and since the photon detector (APD) is fre- 
quency undiscriminating and cannot distinguish between the narrow-band probe and the wide-band squeezing, we need to avoid the probe beam when measuring the kittens. Therefore we introduced a chopping cycle of the probe beam where it is on for $20 \mu$ s and off for the next $80 \mu \mathrm{s}$, chopped by two double-pass AOMs. While the probe is on we do the phase and cavity locking and shield the APD. While it is off, we uncover the APD and measure the conditional homodyne signals. In the meantime, the locks are kept passively stable.

\subsection{Photon subtraction}

The squeezed light impinges on a $5 \%$ reflection beamsplitter, where the main transmitted part goes directly to the homodyne detector for output state analysis. The small reflected part is directed to an avalanche photo diode (APD), whose click signal is connected to a digital oscilloscope to act as a conditioning trigger of the homodyne data acquisition. The squeezed light does not contain many photons, only $5 \%$ is tapped for trigger detection, and the total transmission and detection efficiency of the trigger channel is only about $10 \%$, so the photon count rate is rather low - around 10,000 counts/sec for $3 \mathrm{~dB}$ squeezing. But whenever a photon is detected, we know that it is missing from the correlated part of the main signal beam, which is therefore projected to a photon-subtracted squeezed vacuum state. The low efficiency of the trigger channel only influences the success rate of the experiment; the impact on the generated states is almost negligible.

An APD and a homodyne detector have completely different spectral and temporal mode resolutions, so the photon detected by the APD is not necessarily correlated with the mode seen by the homodyne detector. That is true for both a pulsed laser setup [10] and for a cw setup [11], [12], but the problem is manifested in different ways and requires different solutions. In the $\mathrm{cw}$ case, the down-conversion process happens over a nanometer-broad spectral range, but only at those frequencies resonant with the OPO cavity. The APD can detect all the down-converted photons, but the homodyne detector is limited to a bandwidth of around $50 \mathrm{MHz}$ around the carrier frequency of the local oscillator, derived from the main $860 \mathrm{~nm}$ laser. Therefore we have to eliminate all the non-degenerate OPO spectral cavity modes from the light reaching the APD. We do that by passing the light through two subsequent Fabry-Perot cavities, both resonant with the degenerate OPO mode but with different, rather wide free spectral ranges. This arrangement works very well and seemingly filters away all unwanted photons.

\subsection{Homodyne tomography}

To measure the Wigner functions of the generated non-classical light states, we interfere them with a strong local oscillator (LO) on a 50:50 beam splitter, detect the two output beams on fast PIN photo diodes and monitor the amplified difference photocurrent on the oscilloscope triggered by the APD signal. For every trigger click, we record a trace of about $1 \mu$ s length around the trigger time at a sample rate faster than twice the detector bandwidth. Later, in post-processing, we perform a temporal mode selection by weighting each trace with a mode function

$$
f(t)=\kappa e^{-\gamma\left|t-t_{0}\right|}-\gamma e^{-\kappa\left|t-t_{0}\right|},
$$

where $\gamma$ is the OPO HWHM bandwidth, $\kappa$ the filter cavity bandwidth, and $t_{0}$ the time of photon detection. This mode function corresponds to the temporal extent of the correlations between the detected photon and the photon-subtracted main beam, so it is close to the optimal temporal filter for extracting the conditionally prepared state with high efficiency.

The homodyne detection process described here results in a single outcome of the quadrature measurement at the given LO phase. To reconstruct the full state, we repeat the measurement many thousand times for identically prepared states, with the LO phase locked to one of 6 or 12 evenly distributed values between 0 and $\pi$. Finally, we process the total set of quadrature measurements with a maximum likelihood algorithm [44], [49] to estimate the density matrix of the generated state. An example of a full tomographic measurement along with the reconstructed state (Wigner function calculated from the density matrix) is presented in Fig. 2.
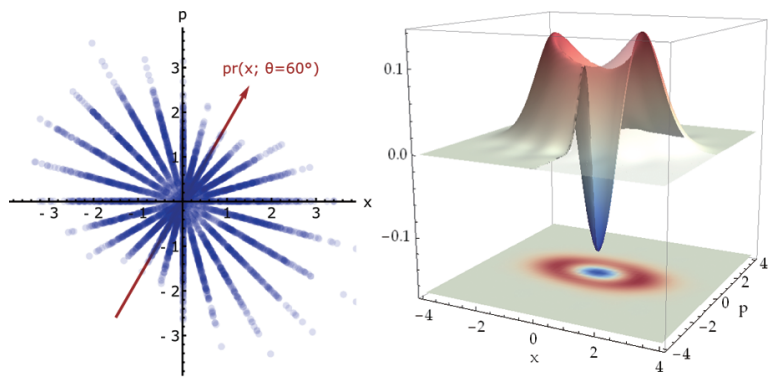

Fig. 2 Example of homodyne tomography of a singlephoton subtracted squeezed vacuum state. On the left is a series of homodyne measurements taken at 12 different fixed phase space angles. Notice the relative lack of observations around the origin - this is reflected in the negative dip of the reconstructed Wigner function in the right panel. The Wigner function has been rotated in phase space to have squeezing along the p-axis. 


\section{Distillation of gaussian entanglement}

One of the great advantages of cv quantum information processing is that it is essentially constructed from deterministic, Gaussian operations, that is, operations with Hamiltonians that are at most quadratic in the field operators. All of the most commonly used techniques in the lab such as squeezing, phase shifting, beam splitting, displacement and homodyne detection (Fig. 3) are Gaussian operations, which means that an input state with Gaussian Wigner function (or characteristic function) remains Gaussian after the transformation. The theory for Gaussian states, entanglement, operations and so on is very well developed and provides a powerful framework for various quantum information processing tasks [50], [51]. Unfortunately, there are some significant exceptions to what is possible with Gaussian resources. Bartlett et al. [52] showed that a quantum computer using only Gaussian resources can be efficiently simulated by a classical computer. Therefore non-Gaussian operations are needed to achieve a quantum-mechanical speed-up of computing. Another problem is long-distance distribution of entanglement which is needed for quantum communication protocols. In order to combat propagation losses, the entanglement must be reinforced by quantum repeater stations [53], incorporating entanglement swapping and distillation [54]. However, it was shown that a Gaussian entangled state cannot be distilled by Gaussian local operations and classical communication [55]-[57]. Since Gaussian entanglement is the predominant type of $\mathrm{cv}$ entanglement (such as two-mode squeezed EPR states), non-Gaussian operations are required for distribution over long distances.

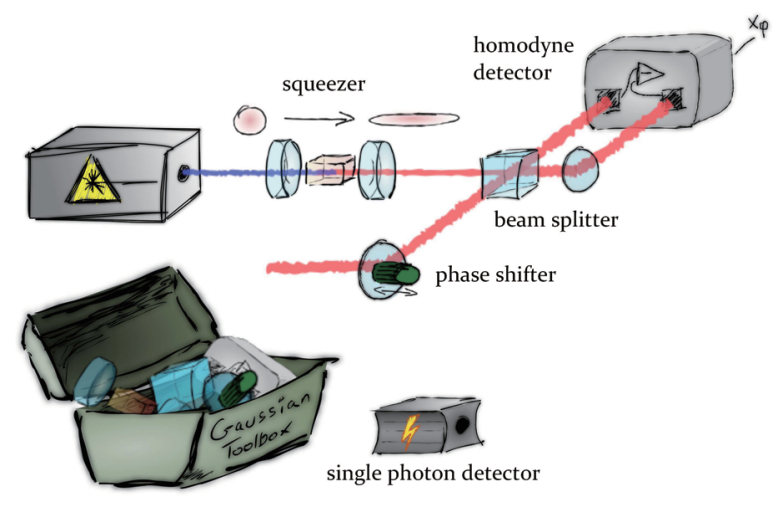

Fig. 3 The Gaussian toolbox. A basic continuous-variable experiment involving the essential Gaussian operations. For a number of important quantum information tasks, this set of Gaussian tools is not sufficient and must be supplemented by a non-Gaussian tool such as a single photon detector.
Hage et al. [58], [59] and Dong et al. [60] performed entanglement distillation using only conditional homodyne detection, but in those cases, the Gaussian entangled beams had been exposed to non-Gaussian noise. Then it is possible to recover the original amount of Gaussian entanglement by Gaussian operations such as homodyne detection. However, the probably most common and critical transmission noise comes from linear loss, which is a Gaussian transformation. Ourjoumtsev et al. [61] demonstrated that a non-local (collective) photon subtraction can increase entanglement that is still Gaussian. Based on this demonstration and on the realization by Opatrný et al. [19] that photon subtraction can increase entanglement and lead to better teleportation, we performed the first entanglement distillation [62] of a Gaussian two-mode state [1].

\subsection{Experiment}

The basic concept of the distillation protocol, illustrated in Fig. 4, is to prepare a series of entangled states shared by Alice and Bob, let them perform local operations on their part and finally let them communicate with each other classically to determine which states have successfully had their entanglement increased and therefore can be kept for further processing. This process sacrifices the rate of shared states for the amount of entanglement.

In our experiment (Fig. 5) we generate a two-mode entangled state simply by splitting a squeezed vacuum of variable squeezing level on a 50:50 beamsplitter. This creates a state with rather weak entanglement, equivalent to that of a standard two-mode squeezed (EPR) state with half as much squeezing. For Alice

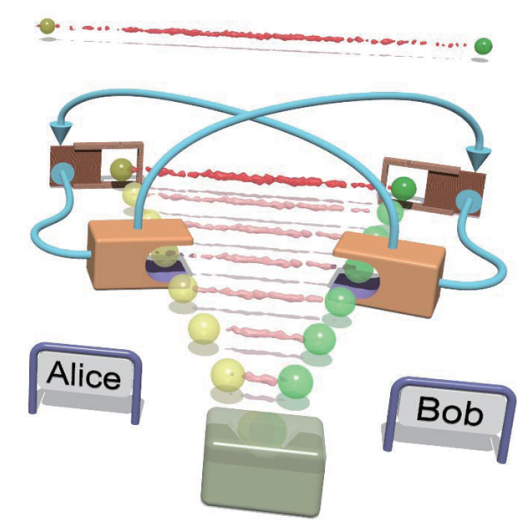

Fig. 4 Basic concept of entanglement distillation. Weak entanglement is enhanced by probabilistic local operations (orange boxes) followed up by classical communication (blue wires) to select those entangled pairs that have been successfully distilled. 


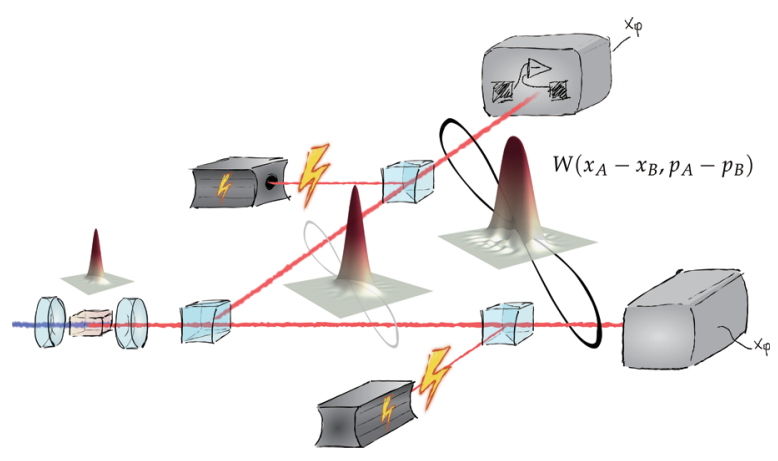

Fig. 5 Entanglement distillation experimental setup, in this case for simultaneous photon subtractions at both Alice and Bob. The Wigner functions illustrate the measured and reconstructed states of the $A-B$ mode. The $A+B$ mode is in all cases vacuum.

and Bob's local operations, we use the photon subtraction technique described in the previous section, with a separate channel of frequency filters and APD for both. A click of the APD detectors signals a successful distillation event. If Alice and Bob were to proceed with an application of their shared entanglement, they would have to classically tell each other at what times they got a detector click. We do not go that far here - instead we simply characterize the resulting quantum state. To quantify the entanglement before and after the distillation, we reconstruct the joint two-mode density matrix by homodyne detection on both output modes for both the undistilled state and for the state with a photon subtracted simultaneously from each of the two modes. It turns out that even subtracting a photon from just Alice or Bob also increases their shared entanglement, so we did a third series of measurements of this scenario. The photon subtraction beamsplitter reflectivities were set at $0 \%$ for the initial state measurement, at $5 \%$ for the single photon detection series and at $10 \%$ for the two simultaneous photon detection series (as a compromise between success rate and entanglement gain).

It is possible to do a complete two-mode tomographic measurement by independently varying the LO phases of Alice's and Bob's homodyne detectors. Fig. 6 shows an example of a density matrix reconstructed from such a measurement, where 36 LO phase combinations $(0-0,0-\pi / 6, \ldots, 0-5 \pi / 6, \pi / 6-0, \ldots, \pi / 6-5 \pi / 6, \ldots$, $5 \pi / 6-5 \pi / 6)$ were used. However, this process is very time-consuming, and due to our particular kind of initial entangled state, the output state can be separated into a vacuum state in the $x_{A}+x_{B}, p_{A}+p_{B}$ variables and a state in the $x_{A}-x_{B}, p_{A}-p_{B}$ variables that corresponds to a photon-subtracted squeezed vacuum. This fact allows us to do the two-mode tomography using

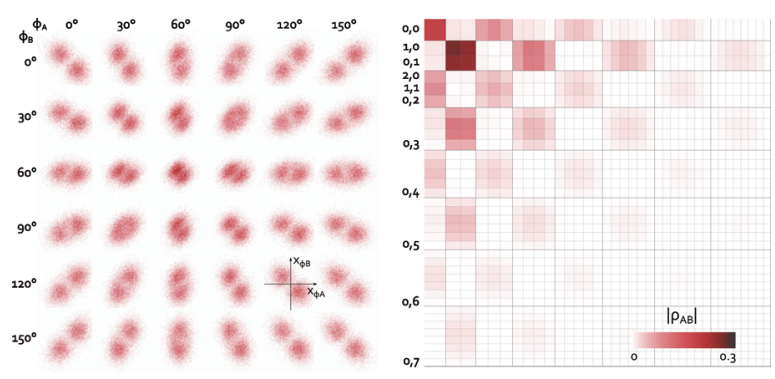

Fig. 6 Full two-mode homodyne tomography of a singleparty distilled state. On the left are the joint quadrature distributions for all 36 combinations of Alice's and Bob's LO phases. From these data the full density matrix (right) was reconstructed. When combining the density matrix elements in groups with the same total photon number, the overall block structure resembles a single mode photonsubtracted squeezed vacuum.

only the 6 phase combinations where the two LO's are in phase, a strategy that is experimentally justified by confirming that the virtual ' + ' mode is indeed in a vacuum state. For details, refer to the Supplementary Information of [1]. In broad terms, the argument relies on the fact that the experimental setup is fully equivalent to a setup where the photon subtraction (by 1 or 2 APDs) is done before the 50:50 beam splitting. That same fact shows that for the single-party distillation operation, the joint output state is independent on whether Alice or Bob detected the photon.

\subsection{Results}

The result of the distillation is shown in Fig. 7a. The entanglement measure we use is the logarithmic negativity [63] which is easily computable from the density matrix. The graph shows that for a wide range of initial squeezing levels, the entanglement gain from the distillation protocol is significant for both singleparty and dual-party operations. Perhaps surprisingly, the single-party distillation mostly works better than the dual-party. This is a result of the particular kind of entangled initial state we use. It can be understood by realizing that for weak squeezing levels, the shared state after a single photon subtraction is essentially a split single photon which has a full e-bit of entanglement, whereas for two photon subtraction (or zero), the shared state is mostly vacuum with a small contribution of photon pairs and therefore with entanglement close to zero. However, the effect of the single-party distillation operation is not limited to this particular scenario - the pure-state calculations in Fig. 7c show that also for the more general two-mode squeezed vacuum state there is a marked increase in entanglement for both single-party 

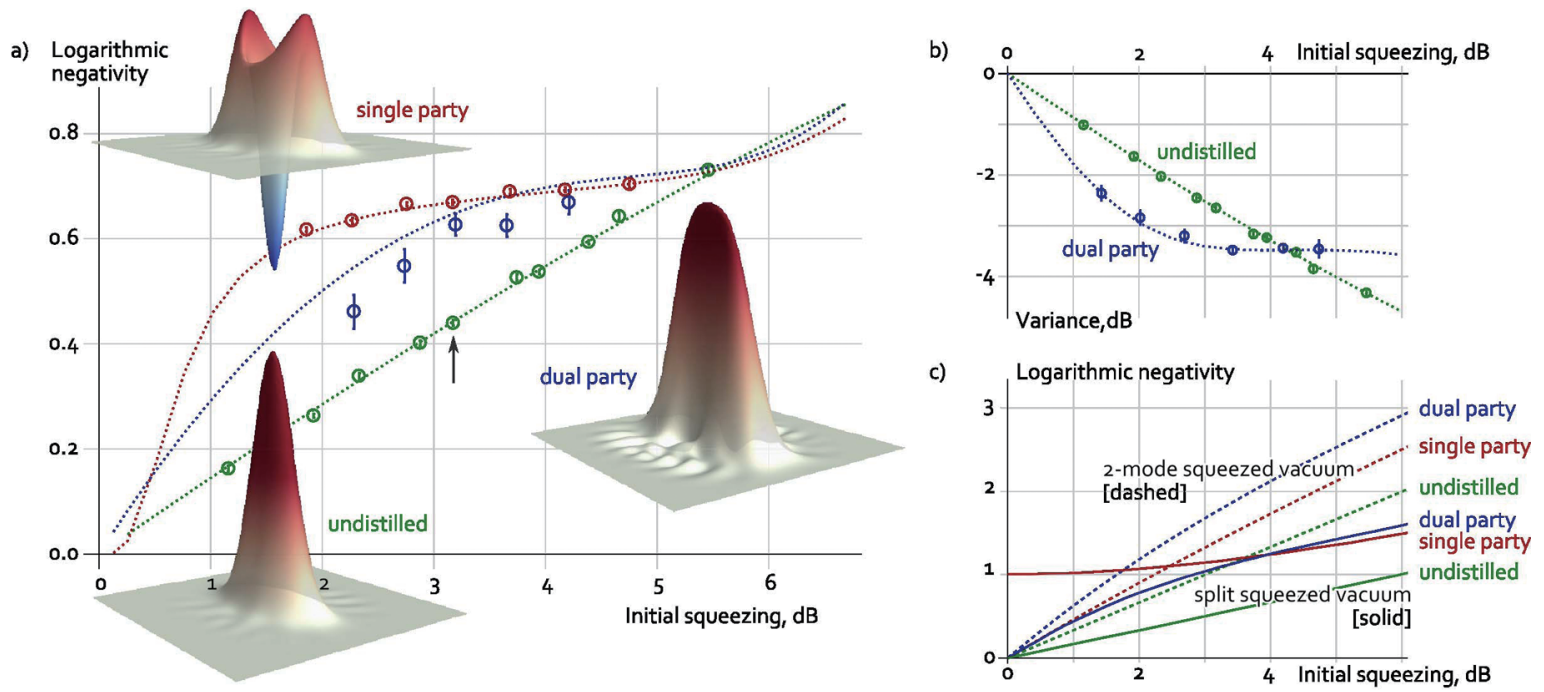

Fig. 7 Entanglement distillation. a) Logarithmic negativity for the undistilled, single-party (1 click) distilled and dual-party distilled states for various input squeezing levels. Dashed curves are calculated from a model taking all experimental parameters into account. The 3D plots are the reconstructed Wigner functions $W\left(x_{A}-x_{B}, p_{A}-p_{B}\right)$ for $3.2 \mathrm{~dB}$ initial squeezing. b) $p_{A}-p_{B}$ quadrature variance for the undistilled and the dual-party distilled states. Because of losses and other imperfections, the undistilled output squeezing is lower than the initial level, whereas the distilled state is actually more strongly squeezed than the initial state. c) Theoretically calculated entanglement negativity for pure states of both 50:50 split squeezed vacuum as used in the experiment and of standard two-mode squeezed vacuum which initially has twice as much entanglement. Both single-party and dual-party photon subtraction are effective for both classes of entangled state.

and dual-party photon subtractions.

For a complete Gaussian input to Gaussian output distillation protocol, the procedure demonstrated here should be expanded to include several similarly prepared states and then be combined with an iterative Gaussification procedure [20]. However, even after just this single step the resulting distilled state is immediately useful for e.g. improvement of teleportation fidelity [19], [64], [65], at least for the dual-party photon subtraction. This can be seen from Fig. 7b, showing that the two-click distilled state not only has higher entanglement, but also a higher degree of squeezing than the input.

\section{Continuous variable qubit states}

The discovery by Knill et al. [66] that quantum computation is possible using only linear optics in combination with single photon sources and photon detectors gave birth to a wealth of research into linear optics quantum computation (LOQC). Specifically, a number of proposals suggested to use coherent states as the basis for circuit-style LOQC [67]-[69]; that is, to encode logical qubits into the physically higher-dimensional coherent states $|\alpha\rangle$ and $|-\alpha\rangle$. It was shown that such schemes could allow efficient computation with only simple linear operations in-line, with the hard operations put off-line to the preparation of the resource states. For large amplitudes $\alpha$ the two states are basically orthogonal, but for small $\alpha$ they have a finite overlap - nonetheless, this scheme is capable of fault-tolerant quantum computing with certain advantages (as well as disadvantages) over traditional single photon-encoded schemes [16]. One particular advantage is - in contrast to the usual two qubit case that all four Bell states can be unambiguously discriminated, leading to higher efficiency for e.g. quantum teleportation [23]. It is also noteworthy that for longdistance optical communication, it has been shown that simple coherent states attain the channel capacity, but the receiver would have to employ collective decoding with a quantum computer for coherent state signals to extract the maximum information [70].

To realize a coherent state-based computing scheme as described here, we need access to resources of arbitrary qubits $a|\alpha\rangle+b|-\alpha\rangle$. The diagonal states with $a=1, b= \pm 1$ (unnormalized) were already approximately realized in the various kitten state experiments mentioned in the introduction, and of course the two basis states are trivial, but all other qubit states still remain to be demonstrated with high fidelities. We suggested a way to accomplish the generation of these complex coherent state superpositions using two-photon subtraction of squeezed vacuum with an added displacement [71]. The displacement before the second photon detec- 

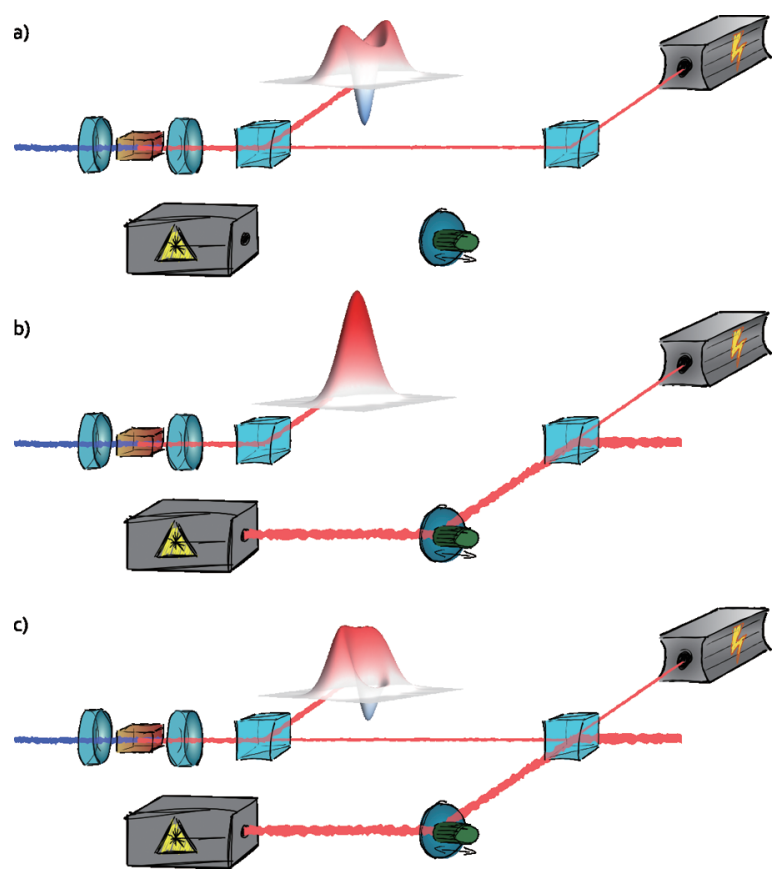

Fig. 8 Superposition state generation by displaced photon subtraction. a) Standard odd kitten state factory. b) With the trigger beam from the squeezing blocked, all photon clicks are from the uncorrelated auxiliary beam. The output state is then just squeezed vacuum. c) With trigger beam and auxiliary displacement beam overlapped on a highly reflective beamsplitter, the two possible sources of the photon detection are indistinguishable and the output is projected to a superposition state determined by the relative strength and phase of the two beams.

tor can be adjusted in phase and amplitude, according to which the conditional output state is prepared in any arbitrary superposition of one-photon and two-photon subtracted states (basically equivalent to a rotation of the coherent state qubit space) as long as $\alpha$ is small.

In our experimental study [2], we demonstrated this method of displaced photon subtraction, although we used only a single photon detector. Our generated states are therefore superpositions of squeezed vacuum and single-photon subtracted squeezed vacuum (equivalent to a squeezed photon). The concept is illustrated in Fig. 8. In a standard kitten state generation setup we insert a highly reflective beamsplitter in the trigger beam just before the APD. On that beamsplitter we inject a strong coherent beam with variable phase and amplitude which acts to displace the state of the trigger beam in phase space. Now the photon detection on the APD becomes ambiguous; there are two possible, indistinguishable sources of the detected photon [72], so the total effect of a click on the trigger beam is a projec-

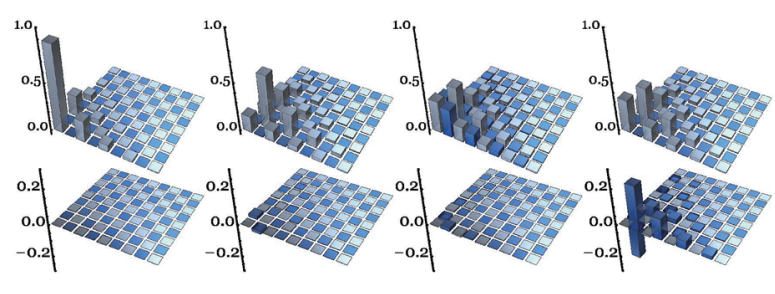

Fig. 9 Fock state basis density matrices of representative reconstructed output states. From left to right: Squeezed vacuum, squeezed photon, a state generated with a displacement beam of $1 / 3$ the intensity (at the APD) of the squeezed trigger beam and with $180^{\circ}$ relative phase, and finally a similar state but with $90^{\circ}$ relative phase. The upper matrices are real parts, lower matrices are imaginary.

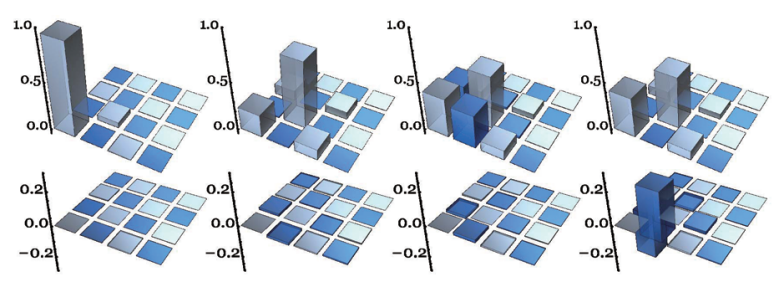

Fig. 10 Squeezed Fock state basis density matrices of the same states as in Fig. 9.

tion onto $\beta|0\rangle+|1\rangle$ and therefore the conditional output state ends up in a superposition of single-photon subtracted squeezed vacuum and normal squeezed vacuum. The exact parameters of the superposition depend on the phase and amplitude of the displacement beam. If the displacement beam is strong (weak), the output will be close to a squeezed vacuum (photon).

To illustrate the potential of this procedure, we carried out a large number of state preparation and characterizations for different displacement parameters. Because we restricted ourselves to single-photon subtraction, we could perform detailed homodyne tomography (360,000 samples at 12 fixed phases) for all these states within a reasonable time span. Fig. 9 shows the density matrices of just a few of these states: squeezed vacuum, squeezed photon, and two states roughly halfway in-between where the trigger and displacement beams were at $180^{\circ}$ and $90^{\circ}$ relative phases. No correction for experimental inefficiencies were performed (and the same goes for the previous distillation results). We have established a detailed model of the full experiment that fits the measured outcomes very well without any free parameters, showing that we have a very good understanding of the individual components of the setup and providing guides to how to improve the results [48].

This experiment truly bridges the $\mathrm{dv}$ and cv worlds. Not only do we use experimental methods from both, 


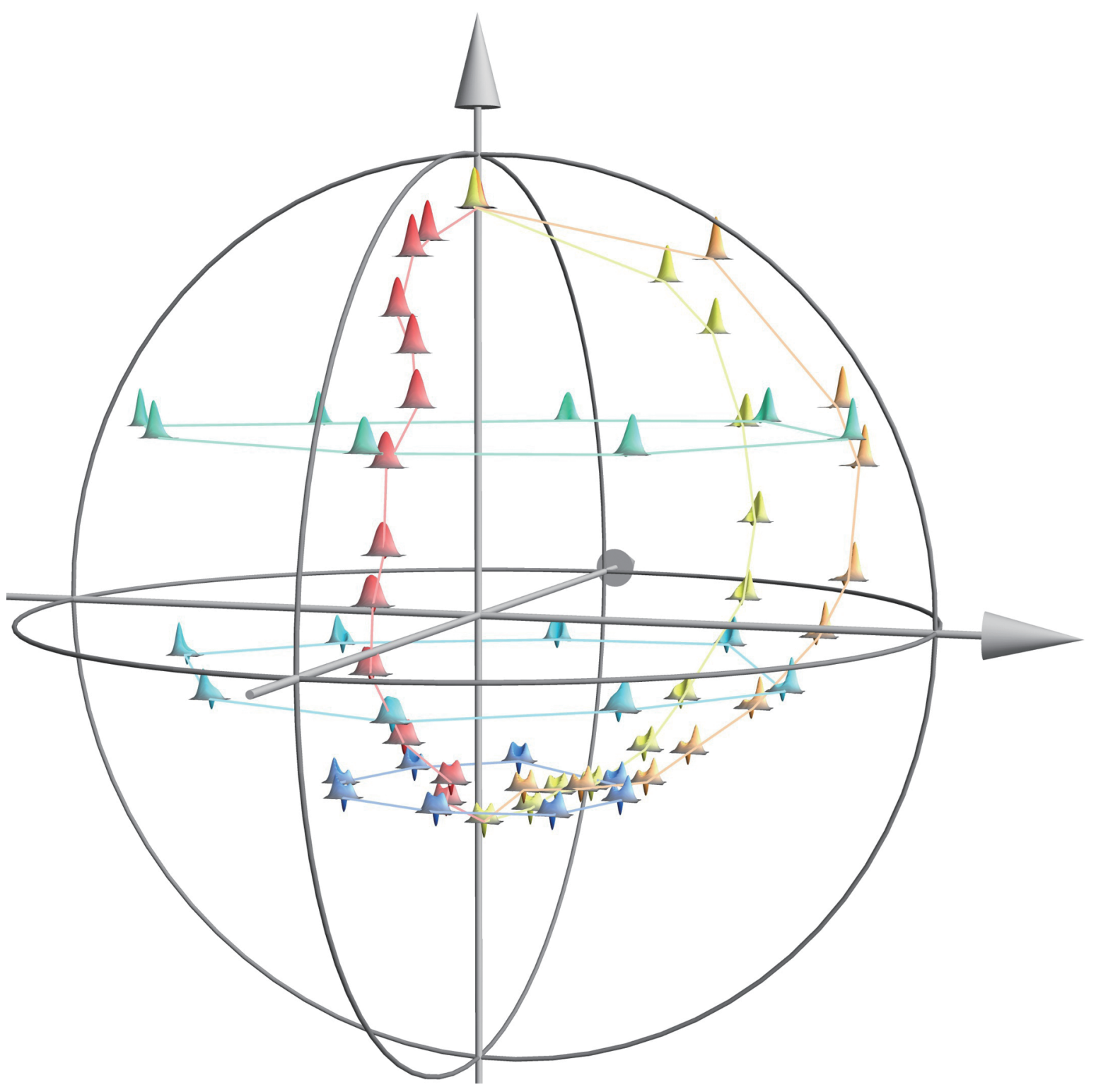

Fig. 11 Bloch sphere with the experimentally generated and reconstructed qubit states plotted by their Wigner functions. The north pole basis state is $\hat{S}(r)|0\rangle$ and south is $\hat{S}(r)|1\rangle$, both with $r=0.38$.

but the output states seen as a whole can also be considered as qubits of squeezed states, being superpositions of the two orthogonal states $\hat{S}(r)|0\rangle$ and $\hat{S}(r)|1\rangle$. To clearly see this, we have transformed the density matrices to a different basis in Fig. 10, namely the squeezed Fock state basis $\left|\phi_{k}\right\rangle=\hat{S}(r)|k\rangle$ - since the squeezing operator is unitary, this is a proper transformation where the new basis is orthonormal and complete. In this squeezed basis, the states are essentially confined to the lowest 2-dimensional subspace $\left\{\left|\phi_{0}\right\rangle,\left|\phi_{1}\right\rangle\right\}$, with a small contribution from higher-order states mostly due to ex- perimental inefficiencies. From these density matrices, we can calculate the parameters for each state when interpreted as a squeezed qubit

$$
|\psi\rangle=\cos \frac{\theta}{2} \hat{S}(r)|0\rangle+e^{i \phi} \sin \frac{\theta}{2} \hat{S}(r)|1\rangle .
$$

For the two non-trivial states of Figs. 9, 10 the parameters are $\theta=100^{\circ}$ and $\phi=0^{\circ}, 90^{\circ}$, respectively. The states are not pure - they range between 0.56 to 0.88 in purity - and therefore the squeezed vacuum and squeezed photon are also not perfectly orthogo- 
nal, with an overlap of 0.26 . The generated qubit states are therefore not directly usable for quantum information purposes as such, but they still serve as a clear demonstration of the potential of the displaced photon subtraction method. In fact, it is also applicable to genuine cat states, since the photon subtraction operation $\hat{a}$ turns the even cat state $|\alpha\rangle+|-\alpha\rangle$ into the odd $|\alpha\rangle-|-\alpha\rangle$ and vice versa, and the displaced photon subtraction can therefore generate an arbitrary superposition of these two states. It was also pointed out by Marek and Fiurášek [73] that the operation corresponds to a single-mode phase gate in the coherent state basis, and that in more complex settings, it can be used to implement the Hadamard gate and a two-mode phase gate, which just serves to emphasize its generally useful quality.

To finish this section and to emphasize the dv-cv link, we present in Fig. 11 the full set of generated qubit states, represented by their reconstructed Wigner functions (cv) and inserted at their appropriate positions in a Bloch/Poincaré sphere (dv) according to the qubit parameters extracted from their squeezed basis density matrices. To see the detailed parameters and close-up Wigner functions of each state, please go to our group's website where an interactive Java applet is available [74].

\section{Conclusion}

We have presented two different examples of what can be accomplished with the photon subtraction operation on non-classical states of light. From a practical applications point of view, it enables a number of different quantum information protocols in continuous variable or hybrid regimes. But it also highlights that the division between the discrete variable and continuous variable worlds is not as large as it was previously made out to be, perhaps to the extent that it hardly makes sense to distinguish them. Judged from the amount of activity on these kinds of hybrid processes and schemes, both on the theoretical and experimental side, we expect to see many more breakthroughs in the near future, ultimately leading to practically applicable quantum information technologies.

\section{References}

[1] H. Takahashi, J. S. Neergaard-Nielsen, M. Takeuchi, M. Takeoka, K. Hayasaka, A. Furusawa, and M. Sasaki, "Entanglement distillation from Gaussian input states," Nature Photonics, vol.4, pp.178-181, 2010.

[2] J. S. Neergaard-Nielsen, M. Takeuchi, K. Wakui, H. Takahashi, K. Hayasaka, M. Takeoka, and M. Sasaki, "Optical continuous-variable qubit," Phys. Rev. Lett., vol.105, 053602, 2010.

[3] U. L. Andersen, G. Leuchs, and C. Silberhorn,
"Continuous-variable quantum information processing," journal Laser \& Photonics Reviews, vol.4, pp.337-354, 2010.

[4] K. Hammerer, A. S. Sørensen, and E. S. Polzik, "Quantum interface between light and atomic ensembles," Reviews of Modern Physics, vol.82, pp.1041-1093, 2010.

[5] D. Bouwmeester, J.-W. Pan, K. Mattle, M. Eibl, H. Weinfurter, and A. Zeilinger, "Experimental quantum teleportation," Nature, vol.390, pp.575-579, 1997.

[6] A. Furusawa, J. L. Sørensen, S. L. Braunstein, C. A. Fuchs, H. J. Kimble, and E. S. Polzik, "Unconditional Quantum Teleportation," Science, vol.282, pp.706-709, 1998.

[7] A. I. Lvovsky, H. Hansen, T. Aichele, O. Benson, J. Mlynek, and S. Schiller, "Quantum State Reconstruction of the Single-Photon Fock State," Phys. Rev. Lett., vol.87, 050402, 2001.

[8] S. A. Babichev, J. Appel, and A. I. Lvovsky, "Homodyne Tomography Characterization and Nonlocality of a Dual-Mode Optical Qubit," Phys. Rev. Lett., vol.92, 193601, 2004.

[9] A. Zavatta, S. Viciani, and M. Bellini, "Quantum-toclassical transition with single-photon-added coherent states of light," Science, vol.306, pp.660-662, 2004.

[10] A. Ourjoumtsev, R. Tualle-Brouri, J. Laurat, and P. Grangier, "Generating optical Schrödinger kittens for quantum information processing," Science, vol.312, pp.83-86, 2006.

[11] J. S. Neergaard-Nielsen, B. M. Nielsen, C. Hettich, K. Mølmer, and E. S. Polzik, "Generation of a Superposition of Odd Photon Number States for Quantum Information Networks," Phys. Rev. Lett., vol.97, 083604, 2006.

[12] K. Wakui, H. Takahashi, A. Furusawa, and M. Sasaki, "Photon subtracted squeezed states generated with periodically poled KTiOPO 4," Optics Express, vol.15, p.3568-3574, 2007.

[13] S. J. van Enk and O. Hirota, "Entangled coherent states: Teleportation and decoherence," Phys. Rev. A, vo.64, 022313, 2001.

[14] A. Gilchrist, K. Nemoto, W. J. Munro, T. C. Ralph, S. Glancy, S. L. Braunstein, and G. J. Milburn, "Schrödinger cats and their power for quantum information processing," J. Opt. B: Quantum and Semiclass. Opt., vol.6, pp.S828-S833, 2004.

[15] H. Jeongand, and T. C. Ralph, "Schrödinger Cat States for Quantum Information Processing," in N. J. Cerf, G. Leuchs, and E. S. Polzik, (editors) "Quantum Information with Continuous Variables of Atoms and Light," Imperial College Press, Chap. 9. 2007.

[16] A. P. Lund, T. C. Ralph, and H. L. Haselgrove, "Fault-Tolerant Linear Optical Quantum Computing with Small-Amplitude Coherent States," Phys. Rev. Lett., vol.100, 030503, 2008. 
[17] M. Dakna, T. Anhut, T. Opatrný, L. Knöll, and D.G. Welsch, "Generating Schrödinger-cat-like states by means of conditional measurements on a beam splitter," Phys. Rev. A, vol.55, pp.3184-3194, 1997.

[18] J. Wenger, R. Tualle-Brouri, and P. Grangier, "NonGaussian Statistics from Individual Pulses of Squeezed Light," Phys. Rev. Lett., vol.92, 153601, 2004.

[19] T. Opatrný, G. Kurizki, and D.-G. Welsch, "Improvement on teleportation of continuous variables by photon subtraction via conditional measurement," Phys. Rev. A, vol.61, 032302, 2000.

[20] D. Browne, J. Eisert, S. Scheel, and M. Plenio, "Driving non-Gaussian to Gaussian states with linear optics," Phys. Rev. A, vol.67, 06232, 2003.

[21] H. Nha and H. Carmichael, "Proposed Test of Quantum Nonlocality for Continuous Variables," Phys. Rev. Lett., vol.93, 020401, 2004.

[22] R. García-Patrón, J. Fiurášek, N. J. Cerf, J. Wenger, R. Tualle-Brouri, and P. Grangier, "Proposal for a Loophole-Free Bell Test Using Homodyne Detection," Phys. Rev. Lett., vol.93, 130409, 2004.

[23] H. Jeong, "Testing Bell inequalities with photonsubtracted Gaussian states," Phys. Rev. A, vol.78, 042101, 2008.

[24] T. Gerrits, S. Glancy, T. Clement, B. Calkins, A. Lita, A. Miller, A. Migdall, S. Nam, R. Mirin, and E. Knill, "Generation of optical coherent-state superpositions by number-resolved photon subtraction from the squeezed vacuum," Phys. Rev. A, vol.82, 031802(R), 2010.

[25] N. Namekata, Y. Takahashi, G. Fujii, D. Fukuda, S. Kurimura, and S. Inoue, "Non-Gaussian operation based on photon subtraction using a photonnumber-resolving detector at a telecommunications wavelength," Nature Photonics, vol.4, p.655-660, 2010.

[26] R. Tualle-Brouri, A. Ourjoumtsev, and P. Grangier, "Quantum Homodyne Tomography of a Two-Photon Fock State," Phys. Rev. Lett., vol.96, 213601, 2006.

[27] H. Takahashi, K. Wakui, S. Suzuki, M. Takeoka, K. Hayasaka, A. Furusawa, and M. Sasaki, "Generation of Large-Amplitude Coherent-State Superposition via Ancilla-Assisted Photon Subtraction," Phys. Rev. Lett., vol.101, 233605, 2008.

[28] A. Ourjoumtsev, H. Jeong, R. Tualle-Brouri, and P. Grangier, "Generation of optical 'Schrödinger cats' from photon number states," Nature vol.448, pp.784786, 2007.

[29] A. Ourjoumtsev, F. Ferreyrol, R. Tualle-Brouri, and P. Grangier, "Preparation of non-local superpositions of quasi-classical light states," Nature Physics, vol.5, pp.189-192, 2009.

[30] A. Zavatta, V. Parigi, M. S. Kim, H. Jeong, and M. Bellini, "Experimental Demonstration of the Bosonic Commutation Relation via Superpositions of Quantum Operations on Thermal Light Fields," Phys. Rev. Lett., vol.103, 140406, 2009.
[31] G. Puentes, J. Lundeen, M. Branderhorst, H. Coldenstrodt-Ronge, B. Smith, and I. Walmsley, "Bridging Particle and Wave Sensitivity in a Configurable Detector of Positive Operator-Valued Measures," Phys. Rev. Lett., vol.102, 080404, 2009.

[32] B. M. Nielsen, J. S. Neergaard-Nielsen, and E. S. Polzik, "Time gating of heralded single photons for atomic memories," Opt. Lett., vol.34, pp.3872-3874, 2009.

[33] E. Bimbard, N. Jain, A. MacRae, and A. I. Lvovsky, "Quantum-optical state engineering up to the twophoton level," Nature Photonics, vol.4, pp.243-247, 2010.

[34] N. Jain, S. R. Huisman, E. Bimbard, and A. I. Lvovsky, "A bridge between the single-photon and squeezedvacuum states," Optics Express, vol.18, 18254, 2010.

[35] A. Zavatta, J. Fiurášek, and M. Bellini, "A highfidelity noiseless amplifier for quantum light states," arXiv:1004.3399 [quant-ph], 2010.

[36] G. Y. Xiang, T. C. Ralph, A. P. Lund, N. Walk, and G. J. Pryde, "Heralded noiseless linear amplification and distillation of entanglement," Nature Photonics, vol.4, pp.316-319, 2010.

[37] F. Ferreyrol, M. Barbieri, R. Blandino, S. Fossier, R. Tualle-Brouri, and P. Grangier, "Implementation of a Nondeterministic Optical Noiseless Amplifier," Phys. Rev. Lett., vol.104, 123603, 2010.

[38] M. A. Usuga, C. R. Mueller, C. Wittmann, P. Marek, R. Filip, C. Marquardt, G. Leuchs, and U. L. Andersen, "Noise-Powered Probabilistic Concentration of Phase Information," Nature Physics, vol.6, pp.767-771, 2010.

[39] C. Wittmann, M. Takeoka, K. N. Cassemiro, M. Sasaki, G. Leuchs, and U. L. Andersen, "Demonstration of near-optimal discrimination of optical coherent states," Phys. Rev. Lett., vol.101, 210501, 2008.

[40] K. Tsujino, D. Fukuda, G. Fujii, S. Inoue, M. Fujiwara, M. Takeoka, and M. Sasaki, "Sub-shot-noise-limit discrimination of on-off keyed coherent signals via a quantum receiver with a superconducting transition edge sensor," Optics Express, vol.18, pp.8107-8114, 2010.

[41] C. Wittmann, U. L. Andersen, M. Takeoka, D. Sych, and G. Leuchs, "Demonstration of coherent-state discrimination using a displacement-controlled photon-numberresolving detector," Phys. Rev. Lett., vol.104, 100505, 2010.

[42] N. Lee, Y. Takeno, H. Benichi, H. Yonezawa, J. Webb, E. Huntington, L. Mišta, R. Filip, P. V. Loock, S. L. Braunstein, and A. Furusawa, "Quantum teleportation of wavepackets in a non-gaussian state. In Conference on Lasers and Electro-Optics/International Quantum Electronics Conference," Optical Society of America, 2009, ITuB4.

[43] M. S. Kim, "Recent developments in photon-level operations on travelling light fields," J. Phys. B: At. Mol. Opt. Phys., vol.41, 133001, 2008. 
[44] A. I. Lvovsky, and M. G. Raymer, "Continuous-variable optical quantum-state tomography," Rev. Mod. Phys., vol.81, pp.299-332. 2009.

[45] P. van Loock, "Optical hybrid approaches to quantum information," Laser \& Photonics Reviews, doi:10.1002/lpor.20100005, 2010.

[46] Y. Takeno, M. Yukawa, H. Yonezawa, and A. Furusawa, "Observation of $-9 \mathrm{~dB}$ quadrature squeezing with improvement of phase stability in homodyne measurement," Optics Express vol.15, pp.4321-4327, 2007.

[47] K. Mølmer, "Non-gaussian states from continuouswave gaussian light sources," Phys. Rev. A, vol.73, 063804, 2006.

[48] M. Takeoka, J. S. Neergaard-Nielsen, M. Takeuchi, K. Wakui, H. Takahashi, K. Hayasaka, and M. Sasaki, "Engineering of optical continuous-variable qubits via displaced photon subtraction: Multimode analysis," $J$. Mod. Opt., doi:10.1080/09500340.2010.533205, 2010.

[49] A. I. Lvovsky, "Iterative maximum-likelihood reconstruction in quantum homodyne tomography," J. Opt. B: Quantum and Semiclassical Optics, vol.6, pp.S556S559, 2004.

[50] P. van Loockand, and S. L. Braunstein, "Quantum information with continuous variables," Rev. Mod. Phys., vol.77, pp.513-577, 2005.

[51] N. J. Cerf, G. Leuchs, and E. S. Polzik, (editors), Quantum Information with Continuous Variables of Atoms and Light Imperial College Press, 2007.

[52] S. D. Bartlett, B. C. Sanders, S. L. Braunstein, and K. Nemoto, "Efficient Classical Simulation of Continuous Variable Quantum Information Processes," Phys. Rev. Lett., vol.88, 097904, 2002.

[53] H.-J. Briegel, W. Dür, J. I. Cirac, and P. Zoller, "Quantum Repeaters: The Role of Imperfect Local Operations in Quantum Communication," Phys. Rev. Lett., vol.81, pp.5932-5935, 1998.

[54] C. Bennett, G. Brassard, S. Popescu, B. Schumacher, J. Smolin, and W. Wootters, "Purification of noisy entanglement and faithful teleportation via noisy channels," Phys. Rev. Lett., vol.76, pp.722-725, 1996.

[55] J. Fiurášek, "Gaussian Transformations and Distillation of Entangled Gaussian States," Phys. Rev. Lett. vol.89, 137904, 2002.

[56] J. Eisert, M. B. Plenio, and S. Scheel, "Distilling Gaussian States with Gaussian Operations is Impossible," Phys. Rev. Lett., vol.89, 137903, 2002.

[57] G. Giedkeand, and J. Ignacio Cirac, "Characterization of Gaussian operations and distillation of Gaussian states," Phys. Rev. A, vol.66, 032316, 2002.

[58] B. Hage, A. Samblowski, J. DiGuglielmo, A. Franzen, J. Fiurášek, and R. Schnabel, "Preparation of distilled and purified continuous-variable entangled states," $\mathrm{Na}$ ture Physics, vol.4, pp.915-918, 2008.
[59] B. Hage, A. Samblowski, J. DiGuglielmo, J. Fiurášek, and R. Schnabel, "Iterative Entanglement Distillation: Approaching full Elimination of Decoherence," Phys. Rev. Lett., vol.105, 230502, 2010.

[60] R. Dong, M. Lassen, J. Heersink, C. Marquardt, R. Filip, G. Leuchs, and U. L. Andersen, "Experimental entanglement distillation of mesoscopic quantum states," Nature Physics, vol.4, pp.919-923, 2008.

[61] A. Ourjoumtsev, A. Dantan, R. Tualle-Brouri, and P. Grangier, "Increasing Entanglement between Gaussian States by Coherent Photon Subtraction," Phys. Rev. Lett., vol.98, 030502, 2007.

[62] We note that there is some confusion about terminology in the literature about entanglement distillation: By some accounts, our result should rather be classified as entanglement concentration, while the term distillation is in line with other sources - notably the conceptually similar procrustean method for qubit distillation of Kwiat et al., Nature 409, 1014 (2001).

[63] G. Vidal and R. F. Werner, "Computable measure of entanglement," Phys. Rev. A, vol.65, 032314, 2002.

[64] P. T. Cochrane, T. C. Ralph, and G. J. Milburn, "Teleportation improvement by conditional measurements on the two-mode squeezed vacuum," Phys. Rev. A, vol.65, 062306, 2002.

[65] S. Olivares, M. G. A. Paris, and R. Bonifacio, "Teleportation improvement by inconclusive photon subtraction," Phys. Rev. A, vol.67, 032314, 2003.

[66] E. Knill, R. Laflamme, and G. J. Milburn, "A scheme for efficient quantum computation with linear optics," Nature, vol.409, pp.46-52, 2001.

[67] P. T. Cochrane, G. J. Milburn, and W. J. Munro, "Macroscopically distinct quantum-superposition states as a bosonic code for amplitude damping," Phys. Rev. A, vol.59, pp.2631-2634, 1999.

[68] H. Jeong and M. S. Kim, "Efficient quantum computation using coherent states," Phys. Rev. A, vol.65, 042305, 2002.

[69] T. C. Ralph, A. Gilchrist, G. J. Milburn, W. J. Munro, and S. Glancy, "Quantum computation with optical coherent states," Phys. Rev. A, vol.68, 042319, 2003.

[70] V. Giovannetti, S. Guha, S. Lloyd, L. Maccone, J. Shapiro, and H. Yuen, "Classical Capacity of the Lossy Bosonic Channel: The Exact Solution," Phys. Rev. Lett., vol.92, 027902, 2004.

[71] M. Takeoka and M. Sasaki, "Conditional generation of an arbitrary superposition of coherent states," Phys. Rev. A, vol.75, 064302, 2007.

[72] The bandwidths of the squeezed vacuum and the coherent displacement beam are quite different, but the very wide integrated bandwidth of the APD erases this source of distinguishability.

[73] P. Marek and J. Fiurášek, "Elementary gates for quantum information with superposed coherent states," Phys. Rev. A, vol.82, 014304, 2010. 
[74] http://www2.nict.go.jp/w/w115/about/53kasai_eng.html

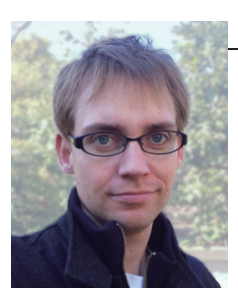

Jonas S. NEERGAARD-NIELSEN Jonas S. NEERGAARD-NIELSEN received his BS and MS degrees in physics from University of Copenhagen, Denmark in 2003 and 2005, respectively. He concluded his $\mathrm{PhD}$ at the Niels Bohr Institute, Univ. of Copenhagen in 2008 and has since then been working at NICT as a limited term researcher. His research in quantum optics and quantum information is focused on generating and manipulating non-classical light, on uniting continuous variable and discrete variable concepts and experimental approaches, and on turning a lot of mirrors.

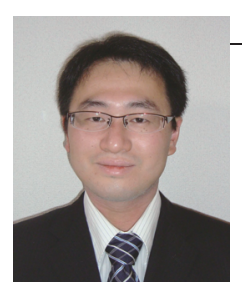

\section{Makoto TAKEUCHI}

Makoto TAKEUCHI received BS in physics from Nagoya University, Japan in 2001. He received MS and $\mathrm{PhD}$ in physics from Kyoto University, Japan in 2003 and 2006, respectively. He joined NICT as a special researcher during 2009-2010. He is currently the assistant professor at the Department of Basic Science, Graduate School of Arts and Sciences, the University of Tokyo. His research interests include quantum states of both light and atomic spin with both cv and dv approaches. Specifically, his experimental skill ranges from homodyne tomography, photon statistics, Faraday rotation and cavity quantum electrodynamics with neutral atoms.

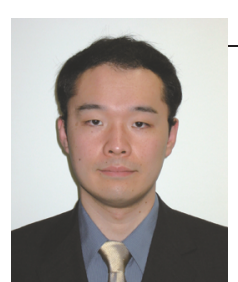

\section{Kentaro WAKUI}

Kentaro WAKUI received a B.S. degree in Electrical Engineering from Waseda University in 2002, and M.S. and Ph.D. degrees in Applied Physics from the University of Tokyo in 2004 and 2007, respectively. $\mathrm{He}$ has worked at Fujitsu Laboratories Ltd. (2008-2009). He currently works at National Institute of Information and Communications Technology in Koganei, Tokyo, where his research topics are related to quantum optics, quantum information processing, and high harmonic generation.

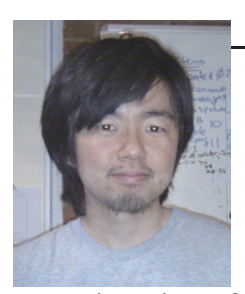

Hiroki TAKAHASHI

Hiroki TAKAHASHI received his bachelor degree from the University of Tokyo in 2004 and Ph.D degree from the same university in 2009. After that he spent a few months as a postdoc in NICT then made a move to University of Sussex in United Kingdom. His current research interest is cavity QED with trapped cold ions.

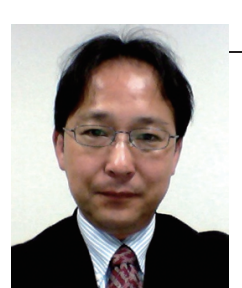

\section{Kazuhiro HAYASAKA}

Kazuhiro HAYASAKA received B.S. and M.S. degrees from the University of Tokyo in 1988 and 1990, respectively. He joined Communications Research Laboratory (reorganized later as National Institute of Information and Communications Technology) in 1990, and received $\mathrm{Ph}$. D degree from Osaka University during his study there. From 1997 to 1998 he was a scientific guest at Max-Planck Institute of Quantum Optics. Since 2007 he has been a visiting associate professor of Osaka University. His research interest includes quantum optics and frequency standards with trapped ions.

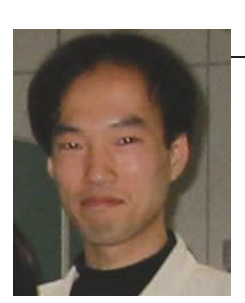

Masahiro TAKEOKA

Masahiro TAKEOKA received the Ph.D. degree in electrical engineering from Keio University in 2001. Since 2001, he has been in National Institute of Information and Communications Technology (NICT) where he is currently a senior researcher and is working on theory and proof-of-principle experiments on quantum detection theory, quantum information theory, and quantum optics. He is a member of the Physical Society of Japan. 


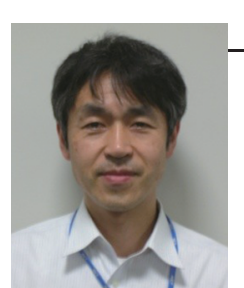

Masahide SASAKI

Masahide SASAKI received the B.S.,

M.S., and Ph.D. degrees in physics from Tohoku University, Sendai Japan, in 1986, 1988 and 1992, respectively. During 1992-1996, he worked on the development of $\mathrm{Si}$ MOSFET with Ayase Laboratory, Nippon Kokan Company, Kanagawa Japan. In 1996, he joined the Communications Research Laboratory, Ministry of Posts and Telecommunications (since 2004, National Institute of Information and Communications Technology, Ministry of Internal Affairs and Communications). Since 1994, he has been working on Quantum Information Theory and Quantum Optics. He is presently group leader of the Quantum ICT group. Dr. Sasaki is a member of Japanese Society of Physics, and the Institute of Electronics, Information and Communication Engineers of Japan. 